\title{
Adaptive Sliding Mode Control of Generator Excitation System with Output Constraints
}

\author{
Liying Sun \\ Electrical Engineering College, Liaoning University of Technology, Jinzhou, Liaoning 121001, China \\ Correspondence should be addressed to Liying Sun; lgsunliying@163.com
}

Received 3 December 2015; Revised 29 May 2016; Accepted 30 May 2016

Academic Editor: Yan-Jun Liu

Copyright (C) 2016 Liying Sun. This is an open access article distributed under the Creative Commons Attribution License, which permits unrestricted use, distribution, and reproduction in any medium, provided the original work is properly cited.

\begin{abstract}
A nonlinear excitation controller for the uncertain parameters and external disturbances is designed by using the backstepping method and sliding mode control theory. In the design process, nonlinear features are retained completely with no linear technique used here. Compared with the excitation controllers designed via conventional linearization, the newly designed one has superiorities in strong robustness to external disturbances, good adaptive ability to variation of system parameters, super transient performance, and the ability to assure preset constraints. Simulation results prove the validity and stability of the controller.
\end{abstract}

\section{Introduction}

Excitation control system has attracted great attention mainly due to its abilities to ensure the stability of power system. Many studies have been focused on the excitation control system and satisfactory results have been obtained in applying theory of differential geometry for precision model to excitation control field of power system [1]. However, with a rapid increase in size and complexity of power system, some parameters in the system are difficult to measure accurately. Additionally, due to the parameter uncertainties of power system, attempt to design a controller by approximating all possible uncertain parameters via appropriate parameter updating law has been proved satisfactory. Various control techniques have been used to develop the nonlinear system [2-6].

The designed controller not only can guarantee the stability of the closed-loop system but also has the adaptive ability to uncertain parameters [7]. Moreover, the excitation control in combination with the coordination control with TCSC can achieve better transient response [8]. Power system is disturbed not only by the internal parameters, but also by external disturbances. How to attenuate the influences of external disturbances must be taken into consideration in power system.
An adaptive robust excitation controller with disturbance suppression not only is applied to solve the internal uncertain parameters and short circuit problems but also can ensure the system stability when subjected to external disturbances $[9$, 10]. Sliding mode control theory can be used to tackle external disturbances of the system, by virtue of which a system switching hyperplane is constructed based on the desired dynamic characteristics. Sliding mode controller makes the state of system converge to the switching hyperplane from outside of the hyperplane. Once the system reaches the switching hyperplane, the controller will enable the system to arrive at origin point along the switching hyperplane. Therefore, this control method has its advantages of fast response speed and strong robustness to various interferences. Excitation controller with sliding mode surface designed in literatures can switch to the ideal control law with the variation of parameters, which greatly enhances the robustness of the system [11, 12]. With the introduction of integral term into the adaptive integral sliding mode controller in the design process, the performance of static and dynamic response will be improved dramatically [13]. The class- $K$ function is added to adaptive variable structure excitation controller in literatures, which can further accelerate the convergence speed in multimachine power systems [14]. However, the constraints in power system are not considered in deriving 
the controller design. In fact, the constraints in the system cannot be avoided [15-17].

With the scale of modern power systems getting larger and larger, more and more types of equipment are applied. Given different operation range of each device, the range of parameter constraints must be considered in the process of designing the controller. For a large class of nonlinear systems, Tee proposed a method, by means of which he designed the constrained nonlinear controller to keep the system output limits to a certain range $[18,19]$. By using this method, the authors designed a constrained excitation controller to realize output constraint of turbo generator unit's power angle $[20,21]$. Variable structure excitation controller designed in literatures can keep the terminal voltage of generator within its limits. But the effects caused by the uncertain parameters of the system are not considered [22]. In this paper, for the generator excitation control system with output constraints, an adaptive sliding mode excitation controller is designed based on the backstepping method and under the conditions that the system has uncertain parameters and external disturbances. The system not only has good adaptive ability to the uncertain parameters and strong robustness to external interferences but also ensures the amplitude constraint of the power angle output of excitation system.

\section{Problem Description}

The single machine infinite bus power system with excitation control system used in this paper is shown in Figure 1. For generator excitation system shown in Figure 1, it can be seen to obtain the stable rotor angle and relative speed by adjusting the excitation control voltage. Mathematical model of the plant is shown in the following:

$$
\begin{aligned}
\dot{\delta}= & \omega-\omega_{0}, \\
\dot{\omega}= & \frac{\omega_{0}}{H} P_{m}-\frac{D}{H}\left(\omega-\omega_{0}\right)-\frac{\omega_{0} V_{s} \sin \delta}{H x_{d \Sigma}^{\prime}} E_{q}^{\prime}, \\
\dot{E}_{q}^{\prime}= & -\frac{1}{T_{d}^{\prime}} E_{q}^{\prime}+\frac{1}{T_{d 0}} \frac{x_{d}-x_{d}^{\prime}}{x_{d \Sigma}^{\prime}} V_{s} \cos \delta+\frac{1}{T_{d 0} d} E_{f} \\
& +d_{1}(t) \\
y= & \delta
\end{aligned}
$$

where $\delta$ is the rotor angle of generator; $\omega$ is the rotor relative speed of generator; $P_{m}$ is the mechanical power of the original motor; $D$ is damping coefficient of the steam turbine generator unit, which is often difficult to measure accurately, and hence $D$ is taken as an uncertain constant parameter; $H$ is the inertia constant of generator rotor; $V_{s}$ is the infinite bus voltage; $E_{q}^{\prime}$ is $q$ axis transient electromotive force of generator; $x_{d}$ is $d$ axis reactance of generator; $x_{d}^{\prime}$ is $d$ axis transient reactance of generator; $x_{q}$ is $q$ axis synchronous reactance of generator; $x_{d \Sigma}^{\prime}=x_{d}^{\prime}+x_{T}+x_{L} / 2 ; x_{q \Sigma}=x_{q}+x_{T}+x_{L} / 2 ; T_{d 0}$ is the time constant of excitation winding; $T_{d}^{\prime}$ is the time constant of excitation winding when the stator winding is closed. Here $T_{d}^{\prime}=T_{d 0} x_{d \Sigma}^{\prime} / x_{d \Sigma} \cdot d_{1}(t)$ is external interference; $\left|d_{1}(t)\right| \leq \rho$,

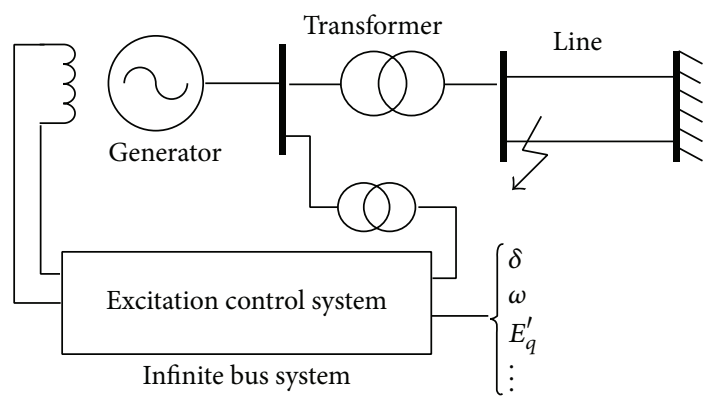

Figure 1: A single machine infinite bus system with generator excitation system.

where $\rho$ is a constant. $E_{f}$ is the excitation control voltage. Let $u$ be input in order to put it clearly; here $u=E_{f} / T_{d 0}$.

Define the new system state variables as $x_{1}=\delta-\delta_{0}, x_{2}=$ $\omega-\omega_{0}$, and $x_{3}=-\omega_{0} V_{s} \sin (\delta) E_{q}^{\prime} /\left(H x_{d \Sigma}^{\prime}\right)$, where $\delta_{0}$ and $\omega_{0}$ are the generator power angle and speed, respectively, when system (1) runs in stable operating point. Let $a_{21}=\omega_{0} P_{m} / H$ and $\theta=-D / H$. $\theta$ is an unknown constant parameter because of $D$ uncertainty. Then, system (1) can be transformed into the following form:

$$
\begin{aligned}
& \dot{x}_{1}=x_{2}, \\
& \dot{x}_{2}=a_{21}+\theta x_{2}+x_{3}, \\
& \dot{x}_{3}=f(x)+g(x) u+d^{\prime}, \\
& y=x_{1}+\delta_{0},
\end{aligned}
$$

where $f(x)=-x_{3} / T_{d}^{\prime}-\omega_{0} V_{s}^{2} \sin \delta\left(x_{d}-x_{d}^{\prime}\right) \cos \delta / H T_{d 0} x_{d \Sigma}^{\prime 2}+$ $x_{2} x_{3} \cos \delta / \sin \delta, g(x)=-\omega_{0} V_{s} \sin \delta / H T_{d 0} x_{d \Sigma}^{\prime}, d^{\prime}=$ $-\left(\omega_{0} V_{s} \sin \delta / H x_{d \Sigma}^{\prime}\right) d_{1}(t)$, and $\left|d^{\prime}\right| \leq h \beta$, where $h$ and $\beta$ are all positive constants.

For system (2), if the absolute value of $x_{1}(0)$ is less than the set positive constant $k_{b}$, then we construct the barrier Lyapunov function through the backstepping method and use estimated value to approach the uncertain parameter $\theta$. Use method of sliding mode variable structure to enhance the robustness of system to external disturbances. The designed controller can guarantee that the rotor angle $y$ belongs to the set $\left(\delta_{0}-k_{b}, \delta_{0}+k_{b}\right)$, which ensures that the output will not break the set constraint.

\section{Controller Design}

Definition 1 (see [18]). A barrier Lyapunov function is a scalar function $V(x)$, defined with respect to the system $\dot{x}=f(x)$ on an open region $D$ containing the origin, that is continuous, is positive definite, has continuous first-order partial derivatives at every point of $D$, has the property $V(x) \rightarrow \infty$ as $x$ approaches the boundary of $D$, and satisfies $V(x(t)) \leq b$, $\forall t \geq 0$, along the solution of $\dot{x}=f(x)$ for $x(0) \in D$ and some positive constant $b$.

Lemma 2 (see [18]). For any positive constants $k_{a 1}$ and $k_{b 1}$, let $Z_{1}:=\left\{z_{1} \in R:-k_{a 1}<z_{1}<-k_{b 1}\right\} \subset R$ and $N:=$ 
$R^{l} \times Z_{1} \subset R^{l+1}$ be open sets. Consider the system $\dot{\eta}=h(t, \eta)$, where $\eta:=\left[w, z_{1}\right]^{T} \in N$ and $h: R_{+} \times N \rightarrow R^{l+1}$ is piecewise continuous in $t$ and locally Lipschitz in $z$, uniformly in $t$, on $R_{+} \times N$. Suppose that there exist functions $U: R^{l} \rightarrow R_{+}$and $V_{1}: Z_{1} \rightarrow R_{+}$, continuously differentiable and positive definite in their respective domains, such that

$$
\begin{aligned}
& V_{1}\left(z_{1}\right) \longrightarrow \infty \text { as } z_{1} \longrightarrow-k_{a 1} \text { or } z_{1} \longrightarrow k_{b 1}, \\
& \gamma_{1}(\|w\|) \leq U(w) \leq \gamma_{2}(\|w\|),
\end{aligned}
$$

where $\gamma_{1}$ and $\gamma_{2}$ are class $K_{\infty}$ functions. Let $V(\eta):=V_{1}\left(z_{1}\right)+$ $U(w)$, and let $z_{1}(0)$ belong to the set $z_{1} \in\left(-k_{a 1}, k_{b 1}\right)$. If the inequality

$$
\dot{V}=\frac{\partial V}{\partial \eta} h \leq 0
$$

holds, then $z_{1}(t)$ remains in the open set $z_{1} \in\left(-k_{a 1}, k_{b 1}\right), \forall t \in$ $[0, \infty)$.

A globally asymptotically stabilizing controller for system (2) will be designed in the following part.

Step 1. Define the error variables $z_{1}=x_{1}, z_{2}=x_{2}-x_{2 d}$, respectively. For the first subsystem of system (2), regarding $x_{2}$ as virtual control, let calm function $x_{2 d}$ be

$$
x_{2 d}=-k_{1}\left(k_{b}^{2}-z_{1}^{2}\right) z_{1},
$$

where $k_{1}$ and $k_{b}$ are positive constants and $\left|z_{1}(0)\right|<k_{b}$. Choose the Lyapunov function of the first subsystem of system (2):

$$
V_{1}=\frac{1}{2} \log \frac{k_{b}^{2}}{k_{b}^{2}-z_{1}^{2}} .
$$

The time derivative of $V_{1}$ along the system trajectory is

$$
\dot{V}_{1}=-k_{1} z_{1}^{2}+\frac{z_{1} z_{2}}{k_{b}^{2}-z_{1}^{2}} \text {. }
$$

Step 2. Define the error variable $z_{3}=x_{3}-x_{3 d}$. For the first two subsystems of system (2), regarding $x_{3}$ as a virtual control, let calm function $x_{3 d}$ be

$$
x_{3 d}=-\frac{z_{1}}{k_{b}^{2}-z_{1}^{2}}-a_{21}-\widehat{\theta} x_{2}+\dot{x}_{2 d}-k_{2} z_{2},
$$

where $k_{2}$ is a positive constant in formula (8), and $\widehat{\theta}=\theta-\tilde{\theta}$ ( $\widehat{\theta}$ is the estimated value of the uncertain constant $\theta . \widetilde{\theta}$ is the estimation error between the true value of $\theta$ and estimated value $\widehat{\theta}$ ).

Augment Lyapunov function of Step 1 as follows:

$$
V_{2}=V_{1}+\frac{1}{2} z_{2}^{2}
$$

The time derivative of $V_{2}$ along the system trajectory is as follows:

$$
\dot{V}_{2}=-k_{1} z_{1}^{2}-k_{2} z_{2}^{2}+z_{2} z_{3}+\tilde{\theta} z_{2} x_{2}
$$

Step 3. It is differentiated for $x_{3 d}$ before designing the global Lyapunov function to extract the estimation error. Consider

$$
\dot{x}_{3 d}=\Phi-\left(\widehat{\theta}+k_{1} k_{b}^{2}-3 k_{1} z_{1}^{2}+k_{2}\right) \tilde{\theta} x_{2} .
$$

Here

$$
\begin{aligned}
\Phi= & -\frac{x_{2}\left(k_{b}^{2}-z_{1}^{2}\right)+2 z_{1}^{2} x_{2}}{\left(k_{b}^{2}-z_{1}^{2}\right)^{2}}-\dot{\hat{\theta}} x_{2}+6 k_{1} z_{1} x_{2}^{2}+k_{2} \dot{x}_{2 d} \\
& -\left(\widehat{\theta}+k_{1} k_{b}^{2}-3 k_{1} z_{1}^{2}+k_{2}\right)\left(a_{21}+\widehat{\theta} x_{2}+x_{3}\right) .
\end{aligned}
$$

Define the sliding surface $\sigma=c_{1} z_{1}+c_{2} z_{2}+z_{3}$, where $c_{1}$ and $c_{2}$ are constants greater than zero. For the entire system (2), augment Lyapunov function of Step 2 by the following:

$$
V_{3}=V_{2}+\frac{1}{2} \sigma^{2}+\frac{1}{2 \gamma} \widetilde{\theta}^{2},
$$

where $\gamma>0$ is a given adaptive gain parameter. Then the time derivative of $V_{3}$ along the system trajectory is as follows:

$$
\begin{aligned}
\dot{V}_{3} & =-k_{1} z_{1}^{2}-k_{2} z_{2}^{2}+z_{2} z_{3}+\widetilde{\theta} z_{2} x_{2}+\sigma\left(c_{1} \dot{z}_{1}+c_{2} \dot{z}_{2}\right. \\
& \left.+\dot{z}_{3}\right)-\frac{1}{\gamma \widetilde{\theta} \dot{\theta}}=-k_{1} z_{1}^{2}-k_{2} z_{2}^{2}+z_{2} z_{3}+\widetilde{\theta} z_{2} x_{2} \\
& +\sigma\left(c_{1} \dot{z}_{1}+c_{2}\left(a_{21}+\widehat{\theta} x_{2}+x_{3}-\dot{x}_{2 d}\right)+f+g u\right. \\
& \left.+d^{\prime}-\Phi\right)+\widetilde{\theta}\left(\sigma c_{2} x_{2}+z_{2} x_{2}\right. \\
& \left.+\sigma\left(\widehat{\theta}+k_{1} k_{b}^{2}-3 k_{1} z_{1}^{2}+k_{2}\right) x_{2}-\frac{1}{\gamma \dot{\dot{\theta}}}\right) .
\end{aligned}
$$

Law of parameter updating is selected as follows:

$$
\dot{\hat{\theta}}=\gamma x_{2}\left(z_{2}+\sigma c_{2}+\sigma\left(\widehat{\theta}+k_{1} k_{b}^{2}-3 k_{1} z_{1}^{2}+k_{2}\right)\right) \text {. }
$$

Control law of excitation voltage input item is as follows:

$$
\begin{aligned}
u= & \frac{1}{g} \cdot\left(-c_{1} \dot{z}_{1}-c_{2}\left(a_{21}+\hat{\theta} x_{2}+x_{3}-\dot{x}_{2 d}\right)-f+\Phi\right. \\
& -h(\sigma+\beta \operatorname{sgn}(\sigma))) .
\end{aligned}
$$

Then

$$
\begin{aligned}
\dot{V}_{3}= & -k_{1} z_{1}^{2}-k_{2} z_{2}^{2}+z_{2} z_{3}-h \sigma^{2} \\
& -\sigma\left(h \beta \operatorname{sign}(\sigma)-d^{\prime}\right) .
\end{aligned}
$$

Because

$$
\begin{aligned}
& z Q z^{T} \\
& =\left[\begin{array}{lll}
z_{1} & z_{2} & z_{3}
\end{array}\right]\left[\begin{array}{ccc}
k_{1}+h c_{1}^{2} & h c_{1} c_{2} & h c_{1} \\
h c_{1} c_{2} & k_{2}+h c_{2}^{2} & h c_{2}-0.5 \\
h c_{1} & h c_{2}-0.5 & h
\end{array}\right]\left[\begin{array}{l}
z_{1} \\
z_{2} \\
z_{3}
\end{array}\right] \\
& =k_{1} z_{1}^{2}+k_{2} z_{2}^{2}-z_{2} z_{3}+h \sigma^{2}
\end{aligned}
$$


select appropriate $k_{1}, k_{2}, c_{1}, c_{2}$, and $h$ to let matrix $Q$ be a positive definite matrix. For the last item $\sigma\left(h \beta \operatorname{sign}(\sigma)+d^{\prime}\right)$ of formula (17), it is positive when $\sigma>0,-h \beta+d^{\prime}>0$. It is still positive when $\sigma<0,-h \beta+d^{\prime}<0$; then $\dot{V}_{3} \leq 0$. We can get the conclusion that the system is asymptotically stable.

So we can get something from the design process above. For system (2), if the initial condition is $z(0) \in \Omega_{z 0}:=\{z(0) \in$ $R^{3}$ and $\left.\left|z_{1}(0)\right|<k_{b}\right\}$ (here, $z=\left[z_{1}, z_{2}, z_{3}\right]^{T}$ ), we can get the following theorems. Please refer to literature [23] for detailed proof.

Theorem 3. Error $z$ always remains in a compact set $\Omega_{z}$ :

$$
\begin{aligned}
\Omega_{z} & =\left\{z \in R^{3}:\left|z_{1}\right| \leq k_{b} \sqrt{1-e^{-2 V_{3}(0)}},\left\|z_{2: 3}\right\|\right. \\
& \left.\leq \sqrt{2 V_{3}(0)}\right\} .
\end{aligned}
$$

Theorem 4. The output $\delta$ always remains in the set $\Omega_{\delta}=\{\delta \in$ $\left.R:|\delta| \leq k_{b} \sqrt{1-e^{-2 V_{3}(0)}}+\delta_{0}\right\}$. That is to say, the output will not go beyond the constraint, and all the closed-loop states of system (5) are bounded.

\section{Simulation Results}

In order to verify the performances of the controller on angle constraint of power systems in this paper, we will compare constraint backstepping method (CBM) proposed in this paper to traditional backstepping method. The design process is the same as the traditional backstepping method (TBM) when the Lyapunov function is set to be a quadratic term form. Please refer to literature [12] for further details. Simulation experiment based on design process above for the single machine infinite bus power system is shown in Figure 1. Parameters are selected as follows:

$$
\begin{aligned}
\delta_{0} & =60^{\circ}, \\
\omega & =314.159 \mathrm{rad} / \mathrm{s}, \\
H & =8 \mathrm{pu}, \\
P_{m} & =0.9 \mathrm{pu}, \\
D & =5 \mathrm{pu}, \\
k_{1} & =1, \\
k_{2} & =1, \\
r & =10, \\
k_{b} & =5^{\circ}, \\
V_{s} & =1 \mathrm{pu}, \\
x_{d} & =2.5 \mathrm{pu}, \\
x_{q} & =2.5 \mathrm{pu}, \\
x_{d}^{\prime} & =0.25 \mathrm{pu},
\end{aligned}
$$

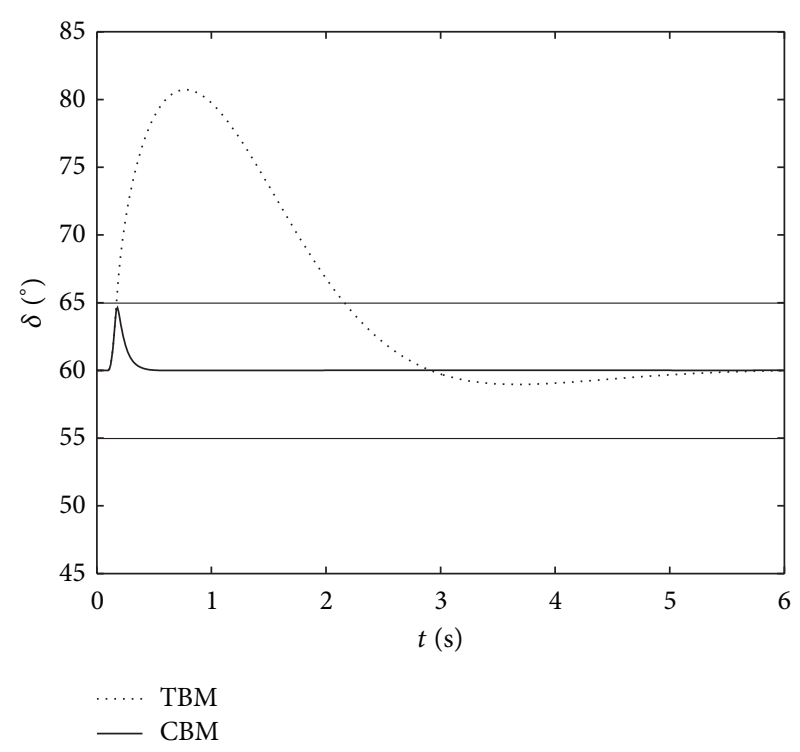

FIgURE 2: Transient response curves of the angle.

$$
\begin{aligned}
x_{T} & =0.1 \mathrm{pu}, \\
x_{L} & =0.3 \mathrm{pu}, \\
T_{d 0} & =12 \mathrm{pu}, \\
c_{1} & =2, \\
c_{2} & =10, \\
h & =50, \\
\beta & =5, \\
d^{\prime} & =0.1 .
\end{aligned}
$$

We can get $\delta<\left|z_{1}\right|+\delta_{0}$ and $\delta_{0}-k_{b}<\delta<\delta_{0}+k_{b}$ from the design process of Section 3. Then $55^{\circ}<\delta<65^{\circ}$.

When the system is in stable operation, response time of fast protection is generally $0.06 \sim 0.15 \mathrm{~s}$ when three-phase short circuit occurs in outlet of the generator near the end line of transformer at the time of $0.1 \mathrm{~s}$. Fault is removed at time $t=0.2 \mathrm{~s}$. Transient responses of the system will go back to normal state shown in Figures 2 and 3.

As we can see from Figure 2, when the system stable operates to $0.1 \mathrm{~s}$, the influences of short circuit make generator torque unbalanced and power angles rise sharply. Short circuit fault is removed and power transmission recovers at $0.2 \mathrm{~s}$. Under the control of constrained sliding mode adaptive controller, power angle will not increase any more and then restore to the original state from $65^{\circ}$. But the traditional methods can not achieve this goal. The power angle will always rise until about $80^{\circ}$. Frequency responses of Figure 3 show that the transient response of constrained sliding mode adaptive method is faster. 


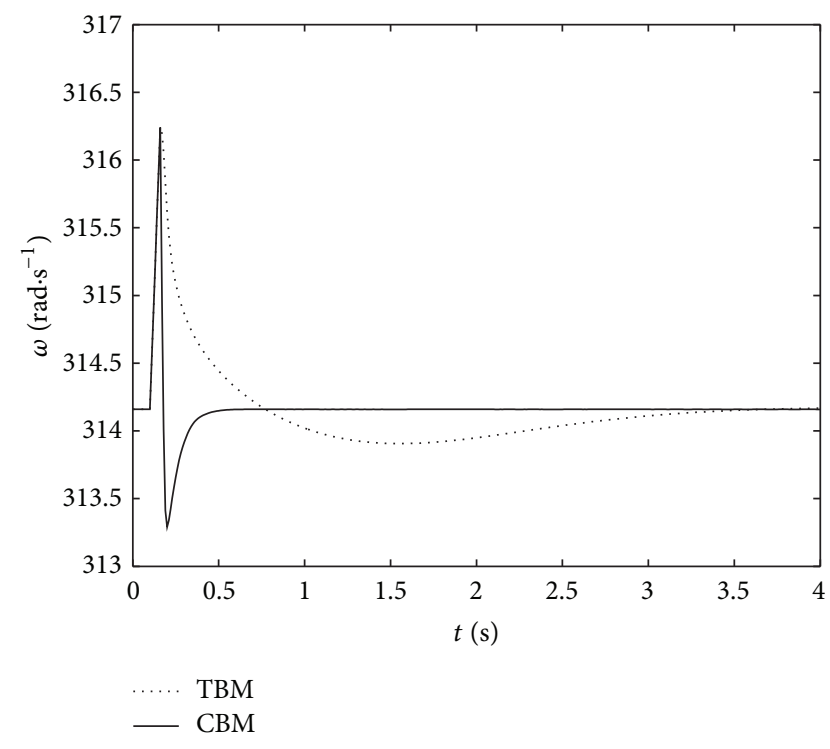

FIgURE 3: Transient response curves of the relative speed.

\section{Conclusions}

Sliding mode adaptive output constraint excitation controller designed in this paper not only can handle uncertain parameters and external disturbances but also can guarantee the preset constraints of the system output. The advantages of the designed controller are summed up as follows: the nonlinear factors of the proposed design are retained. The damping coefficient uncertainty and external interferences are considered in the process of designing. The designed controller has not only strong robustness but also good transient performances. Through the design process and the simulation results above, we can also conclude that as long as the rotor angle $\delta(t)$ belongs to the set $\left(\delta_{0}-k_{b}, \delta_{0}+k_{b}\right)$, the angle of generator will always remain in the range of set value and all the state parameters will be always bounded in the transient process.

\section{Competing Interests}

The author declares that there are no competing interests regarding this paper.

\section{Acknowledgments}

The author gratefully acknowledges the helpful comments and suggestions of the reviewers, which have improved the presentation. Also, thanks go to Yan Zhao and Zhihua Li, their students from the work group, for they have been really involved and helped a lot. Zhao did some simulation work while $\mathrm{Li}$ gave much valuable advice in references and data analysis, and he also formulated the original problem and provided direction and guidance. This work was supported by the National Natural Science Foundation of China (61104070), Natural Science Foundation of Liaoning Province
(2015020076), and Program for Liaoning Excellent Talents in University (LR2013028).

\section{References}

[1] Q. Lu, S. W. Mei, and Y. Z. Sun, Nonlinear Control of Power System, Tsinghua University Press, Beijing, China, 2008.

[2] Y.-J. Liu and S. Tong, "Adaptive fuzzy control for a class of unknown nonlinear dynamical systems," Fuzzy Sets and Systems, vol. 263, pp. 49-70, 2015.

[3] G.-X. Wen, C. L. P. Chen, Y.-J. Liu, and Z. Liu, "Neural-networkbased adaptive leader-following consensus control for secondorder non-linear multi-agent systems," IET Control Theory \& Applications, vol. 9, no. 13, pp. 1927-1934, 2015.

[4] C. L. P. Chen, Y.-J. Liu, and G.-X. Wen, "Fuzzy neural networkbased adaptive control for a class of uncertain nonlinear stochastic systems," IEEE Transactions on Cybernetics, vol. 44, no. 5, pp. 583-593, 2014.

[5] W. He, W. L. Ge, Y. C. Li, Y. J. Liu, C. G. Yang, and C. Y. Sun, "Model identification and control design for a humanoid robot," IEEE Transactions on Systems, Man, and Cybernetics: Systems, 2016.

[6] Y. Gao and Y.-J. Liu, "Adaptive fuzzy optimal control using direct heuristic dynamic programming for chaotic discretetime system," Journal of Vibration and Control, vol. 22, no. 2, pp. 595-603, 2016.

[7] B. H. Wang, C. W. Yang, and Q. Zhang, "Design for adaptive backstepping excitation controller," Transactions of China Electrotechnical Society, vol. 20, no. 12, pp. 91-94, 2005.

[8] L.-Y. Sun, J. Zhao, and G. M. Dimirovski, "Adaptive coordinated passivation control for generator excitation and thyristor controlled series compensation system," Control Engineering Practice, vol. 17, no. 7, pp. 766-772, 2009.

[9] T. L. Shen, S. W. Mei, Q. Lu, W. Hu, and K. Tamura, "Adaptive nonlinear excitation control with L2 disturbances attenuation for power systems," Automatica, vol. 39, no. 1, pp. 81-89, 2003.

[10] L.-Y. Sun, J. X. Feng, G. M. Dimirovski, and J. Zhao, "Adaptive robust $\mathrm{H} \infty$ control of the generator excitation system," in Proceedings of the American Control Conference (ACC '09), pp. 2128-2133, St. Louis, Mo, USA, June 2009.

[11] Y. Ge and C.-W. Li, "Design for $H_{\infty}$ sliding mode robust excitation controller," Proceedings of the Chinese Society of Electrical Engineering, vol. 22, no. 5, pp. 1-4, 2002.

[12] B. H. Wang, "Design of backstepping adaptive sliding mode excitation controller," Electric Power Automation Equipment, vol. 29, no. 6, pp. 54-57, 2009.

[13] X.-Y. Yu, H.-P. Nan, J. Yu, and Y.-X. Huang, "Adaptive integral backstepping sliding mode generator excitation control," Proceedings of the Chinese Society of Electrical Engineering, vol. 29, no. 10, pp. 74-77, 2009.

[14] P.-H. Yang, W.-Y. Liu, Y.-L. Wei, and J.-H. Zhang, "Nonlinear excitation control based on adaptive backstepping and variable structure method," Power System Protection and Control, vol. 40, no. 20, pp. 125-129, 2012.

[15] Y. Liu, Y. Gao, S. Tong, and Y. Li, "Fuzzy approximation-based adaptive backstepping optimal control for a class of nonlinear discrete-time systems with dead-zone," IEEE Transactions on Fuzzy Systems, vol. 24, no. 1, pp. 16-28, 2016.

[16] S. C. Tong, L. L. Zhang, and Y. M. Li, “Observed-based adaptive fuzzy decentralized tracking control for switched uncertain 
nonlinear large-scale systems with dead zones," IEEE Transactions on Systems, Man, and Cybernetics: Systems, vol. 46, no. 1, pp. 37-47, 2016.

[17] G. Y. Lai, Z. Liu, Y. Zhang, C. L. P. Chen, S. L. Xie, and Y. J. Liu, "Fuzzy adaptive inverse compensation method to tracking control of uncertain nonlinear systems with generalized actuator dead zone," IEEE Transactions on Fuzzy Systems, 2016.

[18] K. P. Tee, S. S. Ge, and E. H. Tay, "Barrier Lyapunov functions for the control of output-constrained nonlinear systems," Automatica, vol. 45, no. 4, pp. 918-927, 2009.

[19] K. P. Tee, S. S. Ge, and E. H. Tay, "Adaptive control of a class of uncertain electrostatic microactuators," in Proceedings of the American Control Conference (ACC '07), pp. 3186-3191, New York, NY, USA, July 2007.

[20] Z. H. Li and L. Y. Sun, "Nonlinear control for synchronous generator excitation system with output constraints," Journal of Liaoning University of Technology (Natural Science Edition), vol. 33, no. 3, pp. 141-144, 2013.

[21] S. Liying and L. Zhihua, "Nonlinear adaptive backstepping control for synchronous generator excitation system with output constraints," in Proceedings of the 5th International Conference on Intelligent Control and Information Processing (ICICIP '14), pp. 336-340, Dalian, China, August 2014.

[22] L. Wan, C. H. Deng, and Y. P. Chen, "Multi-layer nonlinear variable structure excitation control considering voltage limitation," Proceedings of the CSEE, vol. 28, no. 19, pp. 86-92, 2008.

[23] K. Wang, H. Xin, D. Gan, and Y. Ni, "Non-linear robust adaptive excitation controller design in power systems based on a new back-stepping method," IET Control Theory and Applications, vol. 4, no. 12, pp. 2947-2957, 2010. 


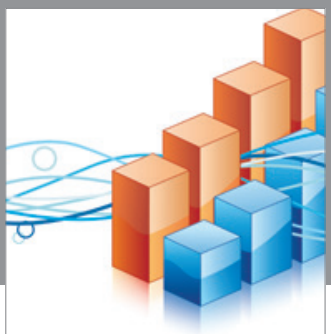

Advances in

Operations Research

vatem alat4

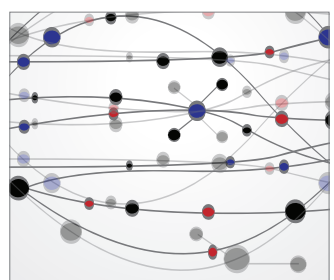

\section{The Scientific} World Journal
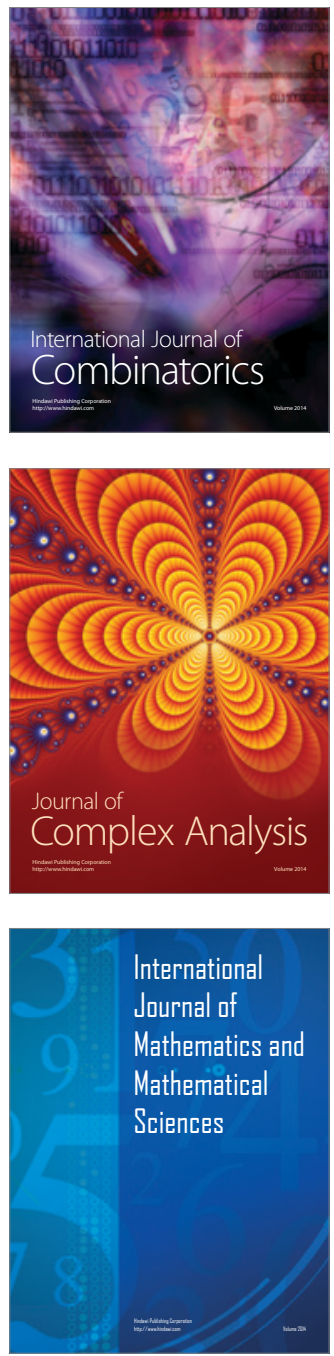
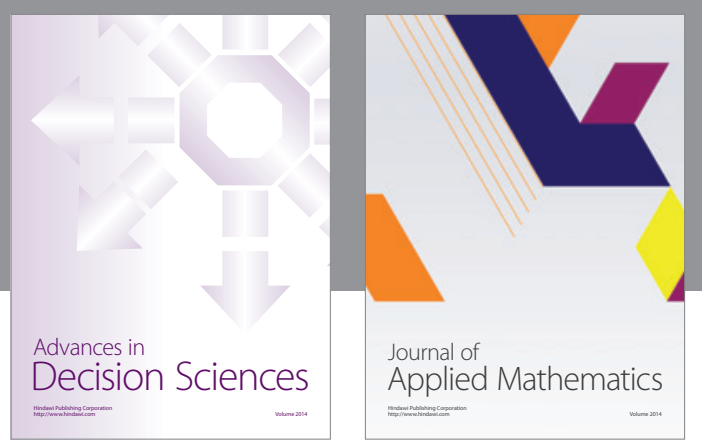

Algebra

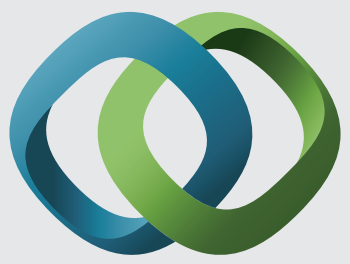

\section{Hindawi}

Submit your manuscripts at

http://www.hindawi.com
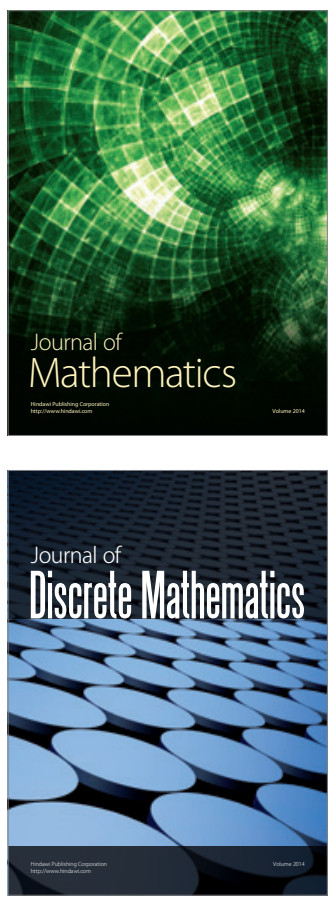

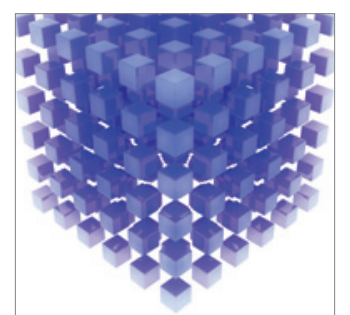

Mathematical Problems in Engineering
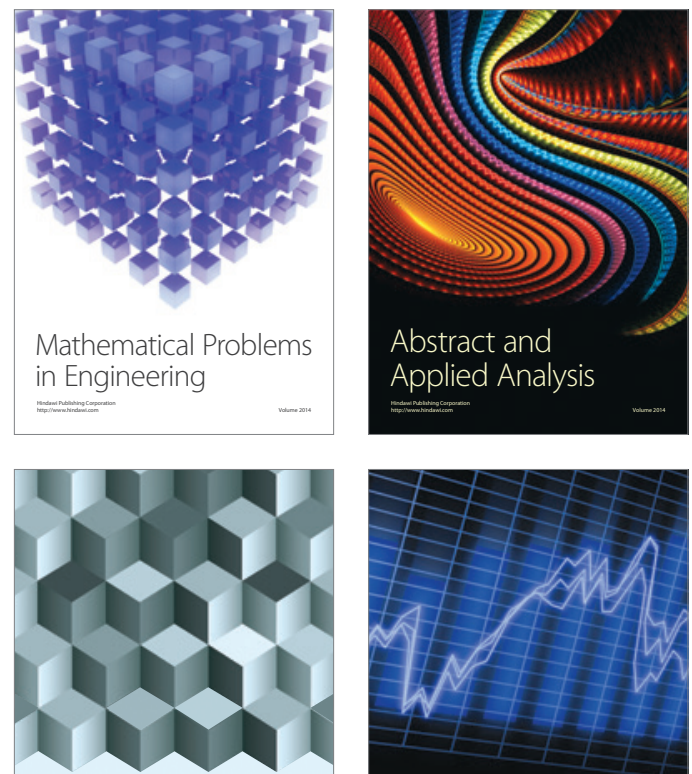

Journal of

Function Spaces

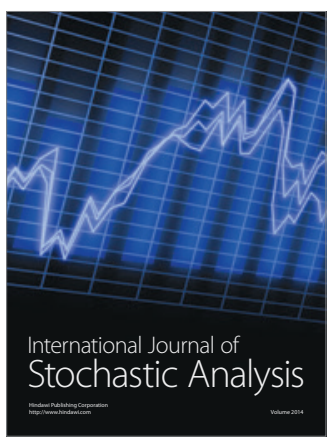

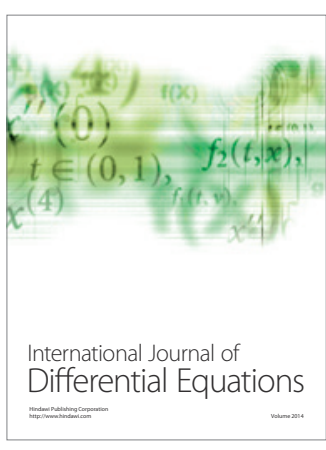
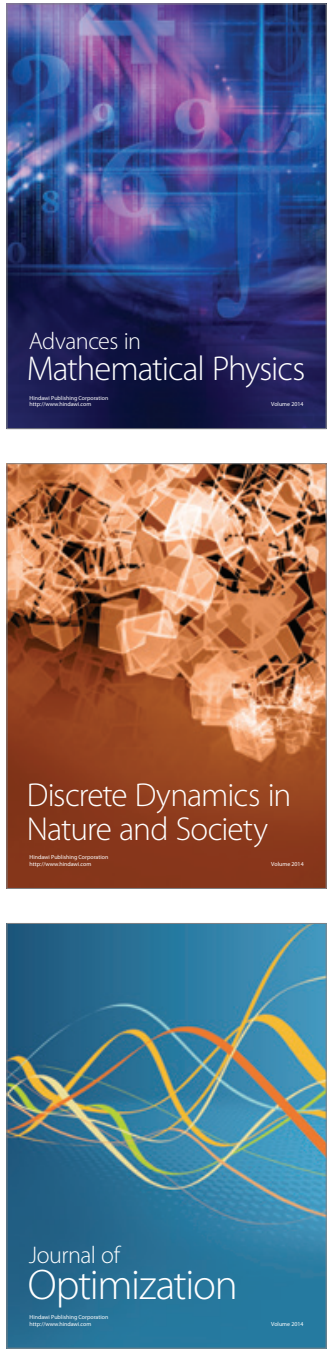